\title{
ORGANIZATIONAL DISCONNECTION AND ITS IMPACTS ON THE EFFECTIVENESS OF PUBLIC CLEANING IN THE MUNICIPALITY OF ALENQUER-PA
}

\section{Marlisson Cleberson Lima Garcia ${ }^{1}$, Madson Alan de Jesus Costa ${ }^{2}$, José Augusto da Costa Colares ${ }^{3}$, Hortência Araújo de Sousa ${ }^{4}$, Marco Aurélio Oliveira Santos ${ }^{5}$}

\author{
1,2, 3,4 Graduando no Curso de Administração da Universidade Federal do Oeste do Pará (UFPA). \\ ${ }^{5}$ Mestre em Agronegócio. Universidade Federal do Oeste do Pará, Campus Alenquer-PA, Brasil \\ Email: marlisson.16garcia@gmail.com, alankosta25@gmail.com, augusto.colares007@gmail.com, \\ hortencia.araujo2011@hotmail.com, marcooliveira_santos@yahoo.com.br.
}

Received: March 26th, 2019

Accepted: May 16 th 2019

Published: June $30^{\text {th }}, 2019$

Copyright (C2016 by authors and Institute of Technology Galileo of Amazon (ITEGAM).

This work is licensed under the Creative Commons Attribution International License (CC BY 4.0). https://creativecommons.org/lice nses/by/4.0/

\begin{abstract}
The study aims to understand how the intraorganizational interactions of the Public Cleaning Coordination in the Municipality of Alenquer-PA influence the effectiveness of the services provided by the coordination. For that, an interview was used with public cleaning coordinator, administrative secretary, class leader and garis. It was observed that due to the lack of effective organizational differentiation and, above all, the lack of coordination and a shared organizational culture between different secretariats and their managers has induced organizational conflicts that limit organizational effectiveness. It is concluded that the lack of coordination by the internal dysfunctions and with the other public sectors of the municipality has provoked inefficiency in the local public services. This fact contributes to a better understanding of the impact of intraorganizational dimensions on the effectiveness of public services.
\end{abstract}

Keywords: Public administration, organizational structure, organizational effectiveness

\section{DESCONEX ̃̃O ORGANIZACIONAL E SEUS IMPACTOS NA EFETIVIDADE DA LIMPEZA PÚBLICA NO MUNICÍPIO DE ALENQUER-PA}

\section{RESUMO}

O estudo tem o intuito de entender como as interações intraorganizacionais da Coordenadoria de Limpeza Pública no Município de Alenquer-PA influenciam a efetividade dos serviços prestados pela coordenadoria. Para tanto, utilizou-se de entrevista com coordenador de limpeza pública, secretário administrativo, chefe de turma e garis. Foi observado que devido à falta de efetiva diferenciação organizacional e, sobretudo, pela falta de coordenação e de uma cultura organizacional compartilhada entre diferentes secretarias e seus gestores têm induzindo conflitos organizacional que limitam a efetividade organizacional. Conclui-se que a falta de coordenação, pelas disfunções internas, com os demais setores públicos do município tem provocado ineficiência nos serviços públicos local. Fato que contribuir para um melhor entendimento do impacto das dimensões intraorganizacionais para efetividade dos serviços públicos.

Palavras chaves: Administração pública, estrutura organizacional, efetividade organizacional 


\section{INTRODUÇÃO}

O lixo é um problema que afeta a qualidade de vida das pessoas e de outros seres vivos que habitam o planeta. Os impactos negativos ao meio ambiente variam desde a contaminação de corpos d'água e do solo à poluição do ar e do visual entre outros tipos de contaminação ambiental. O lixo descartado se torna abrigo para transmissores de doenças como insetos e roedores responsáveis por doenças como febre tifoide, cólera, amebíase, disenteria, giardíase, ascaridíase, leishmaniose, febre amarela, dengue, malária, leptospirose e peste bubônica [1] [2].

A limpeza pública é um fator de importância para sobrevivência humana, pois reduz a incidência de inúmeros vetores que promovem a insegurança humana [3-5].O estado da arte da pesquisa em segurança humana migrou dos efeitos catastróficos originado por armas, para o conjunto de vetores que colocam a vida humana em risco [4] [6]. Assim quando pensamos em limpeza pública pensamos no combate a um conjunto de eventos cotidianos que colocam a vida humana em risco.

Em se tratando de limpeza pública Estado do Pará apresenta um dos mais baixos níveis de domicílios atendidos regularmente pelo sistema de coleta de lixo. Os municípios paraenses apresentam percentuais de domicílios com coletas de lixo heterogênea, figura 1. Pesquisas de conclusão de curso do Plano Nacional de Formação de Professores da Educação Básica (PARFOR) da Universidade Federal do Oeste do Pará (UFOPA) tem apontado problemas quanto a gestão dos resíduos sólidos na cidade de Alenquer-PA [79].

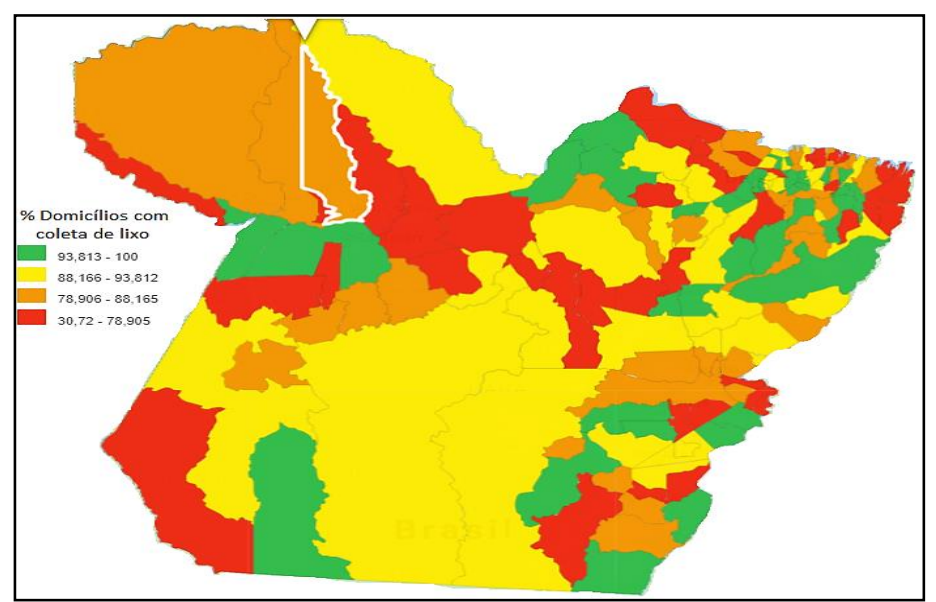

Figura 1: Porcentagem de domicilio paraense com coleta de lixo. Fonte: Atlas, (2010).

Pelas pesquisas do PARFOR se observa que uma parcela da população está sujeita a diferentes tipos de vulnerabilidade sociais exprimindo a situação de fragilidade. Entretanto, o município se encontra em entre aqueles com maior taxa de domicílios com atendimento de coleta de lixo. Cerca de $82 \%$ dos domicílios são atendidos pela Coordenadoria de Limpeza Pública. Esse cenário evidencia gap na efetividade dos serviços prestados pela coordenadoria evidenciando falhas entre o que foi planejado e os resultados alcançados [11][12], mesmo que as decisões sejam tomadas por atores racionais cercados por informações [13].

Para mitigar esses problemas os municípios necessitam contar com uma estrutura organizacional efetiva que possa diminuir situações que colocam a vida humana em risco. Porém, muitas vezes a diferenciação organizacional esbarra no jogo de interesse que dificulta a cooperação políticas nas diferentes arenas decisórias, limitando a construção de uma agenda de política pública voltada para educação, saúde, segurança, moradia, emprego etc., que afetará diretamente as condições de bem-estar da sociedade [14-16]. O resultado é uma entrega de serviços não condizentes com as reais necessidades da população.

Para superar limitantes ao serviço público, faz-se necessárias práticas mistas (intra e interorganizacional) dotadas de capacidades [17-19], para analisar e tomar decisões, bem como partilhar informações e conhecimentos de modo coordenado para minimizar a incidência de problemas oriundos dos resíduos sólidos. Sendo que, as capacidades organizacionais estão ligadas ao desenvolvimento de recursos internos que favoreçam a interrelação entre os diferentes atores [20-22].

Se as nações desejam desenvolver capacidades, elas devem fazer mais do que expandir habilidades humanas individuais. Elas também precisam criar as oportunidades e os incentivos para que as pessoas usem e estendam essas habilidades em prol do desenvolvimento sustentável. O desenvolvimento sustentável segundo a Organização das Nações Unidas (ONU) é: “o desenvolvimento capaz de suprir as necessidades da geração atual, sem comprometer a capacidade de atender as necessidades das futuras gerações" [23-27]. Assim desenvolver capacidades no setor de limpeza pública é importante para alcançar melhor qualidade de vida.

Para tanto é relevante a consolidação de boas práticas organizacionais para o melhor desempenho dos serviços públicos. Assim é importante que os aparelhos estatais sejam agrupados e coordenados de acordo com as relações de responsabilidades, de autoridade, de comunicação e de decisões de uma organização [2831]. Sabe-se que um bom funcionamento da estrutura organizacional é de importância para as organizações públicas ou privadas no processo de entrega de bens e serviços.

Há diversos conceitos sobre o que seria uma estrutura organizacional. Para [32] a estrutura organizacional é:

O resultado de um processo no qual à autoridade é distribuída, as atividades são especificadas (desde os níveis mais baixos até a alta administração) e um sistema de comunicação delineado, permitindo que as pessoas realizem as atividades e exerçam a autoridade que lhes compete para o alcance dos objetivos da organização [32].

A estrutura organizacional é: "a disposição e a inter-relação entre as partes, componentes e cargos de uma empresa" [30]. Na realidade, compreende a disposição das unidades que compõem a empresa (departamentos, divisões, seções etc.), bem como as relações entre superiores e subordinados e abrange os deveres, as responsabilidades, os sistemas de autoridade e de comunicações existentes [28][30]. Assim a estrutura organizacional é um complexo sistema de relações entre indivíduos, grupos e unidades organizacionais orientados por um sistema de valores compartilhados pelos membros que diferenciam uma organização das demais.

Os sistemas de valores, ou a cultura organizacional, evidenciam que as organizações são sistemas socialmente construídos. Como a cultura organizacional é formada a partir da lente interpretativa dos indivíduos que a compõe pode haver relações de cooperação ou conflito fato que tem forçado os gestores a reconstruir suas organizações [28] [30] [31] [33]. Assim entender como os indivíduos, e sobretudo os burocratas com função gerencial, constroem a realidade é entender padrões estruturais de interações e como essas interações impactam na efetividade das organizações.

Analisar as interações intraorganizacionais é um caminho para entender a efetividade organizacional, a comunicação entre os integrantes, as relações formais e informais, assim como outros tipos de relações. $\mathrm{O}$ estudo tem o intuito entender como as interações intraorganizacionais da COLIMP influenciam a 
efetividade dos serviços prestados pela coordenadoria. Com isso, espera-se contribuir com a identificação de falhas organizacionais que devem ser mitigadas para que haja um melhor funcionamento deste departamento governamental. Além dessa introdução o trabalho apresenta os materiais e métodos, os resultados e discussão e as considerações finais.

\section{METODOLOGIA}

O ensejo desta pesquisa foi levantar informações que propiciassem entender a eficiência de uma agência governamental em produzir bens e serviços. Para tanto, utilizou-se de entrevistas nos diversos setores da coordenadoria de limpeza pública do município de Alenquer-PA, COLIMP, para entender a diferenciação organizacional, a cultura organizacional e suas relações intraorganizacionais e como elas impactam na entrega de bens e serviços aos atores do ambiente externo. As entrevistas foram transcritas e analisadas por meio da análise de conteúdo [34]. Participaram da mesma o coordenador de limpeza pública, secretário administrativo, chefe de turma e gari. Através deste, foi possível perceber qual a realidade deste setor no município, enfatizando os problemas, enfrentados, e os planos futuros para um melhor funcionamento desta coordenadoria.

\section{RESULTADOS E DISCUSSÕES}

\section{III.1 A IMPORTÂNCIA DA ESTRUTURA ORGANIZACIONAL PARA O SETOR PÚBLICO}

Com o aumento populacional e tecnológico, tornou-se muito mais complexa a forma de lidar com o ambiente interno e externo das organizações. Com o surgimento da era moderna aumentou-se as necessidades humanas, tanto no contexto material, quanto no psicológico. E assim cabe às organizações adaptar-se para atender às complexidades do homem moderno.

Com as contribuições feitas por autores da administração, foi possível se ter organizações que atendam às diversas necessidades dos indivíduos que recebem os produtos ou serviços prestados. O modelo de estrutura organizacional utilizado pelas indústrias foi e é utilizado e adaptado a diversos setores até os dias de hoje. Na área do setor público a estrutura deveria ser adaptada aos objetivos organizacionais de modo a alcançar uma melhor organização racional do trabalho por meio da divisão de cargos e tarefas, e da especialização do indivíduo no cargo.

Para se suprir as necessidades da população, o setor público aderi a modelos de departamentalização, com a finalidade de que os setores estejam interligados formando uma rede de comunicação, entre as diversas secretarias ou setores, organizando de forma racional os serviços prestados à população sendo possível trazer a máxima produtividade, através da divisão de trabalho, competência técnica, caráter formal das comunicações, hierarquia de comando, impessoalidade, adequação às normas e cultura organizacional. com todos esses fatores o trabalho do setor público se torna efetivo e eficaz, facilitando o trabalho do gestor principal, dando a ele uma visão geral da organização, através do organograma. Porém em muitos casos há disfunções. Devido a um padrão de serviço que gera mudanças, que por seu turno gera resistência.

A princípio as ideias de burocracia criadas por Max Weber, geram uma organização racional do trabalho, mostrando de forma clara e concreta os diversos processos do trabalho realizado pelas organizações, gerando uniformidade das rotinas e procedimentos. No entanto em muitos casos, a burocracia se torna um gargalo aos indivíduos que recebem o serviço prestado, devido as disfunções encontradas nos processos burocráticos.
Esse são fatores comumente vem a acontecer no setor público em razão da falta de profissionalismo e/ou especialização dos ocupantes dos cargos públicos. Apesar dos problemas que comumente vem a acontecer, a burocracia é muito importante para uma estrutura organizacional.

Para se ter uma boa estrutura organizacional, é importante atentar ao lado formal citado acima, e ao lado informal, já que o setor público lida diretamente com pessoas. O lado formal garante o controle dos procedimentos, já o lado informal diz respeito a uma melhor comunicação e a motivação dos agentes organizacionais. Como o ser humano não é motivado unicamente por valores materiais, mas também por valores emocionais os grupos ganham notoriedade no processo de desenvolvimento organizacional. Nesses grupos a cultura organizacional, os interesses e os conflitos se tornam latentes. Cabe ao gestor a ser um mediador do jogo político, pois esse se torna um aspecto essencial da vida organizacional.

Então, observa-se que as capacidades dos gestores públicos são estritamente necessárias, para a eficácia das diversas atividades referentes ao serviço público, pois através de regras e ações eficientes, se tem uma orientação dos requisitos a serem atendidos para se ter uma melhor qualidade no serviço prestado à população.

\section{III.2 CARACTERÍSTICAS DA COORDENADORIA DE LIMPEZA PÚBLICA}

A coordenaria de limpeza pública do município de Alenquer (COLIMP), que é fixada à secretaria municipal de infraestrutura (SEMINF), possui atualmente um coordenador, que atua há um ano e oito meses na gerencia desse órgão público. $\mathrm{O}$ mesmo possui apenas o ensino médio completo. $\mathrm{O}$ mesmo foi alçado ao cargo por meio da indicação do prefeito municipal.

Atualmente a COLIMP possui 142 funcionários no total, sendo 1 coordenador, 2 secretários, 3 chefes de turma e 136 garis, sendo esses divididos em: varredores de rua, limpadores de bueiros, roçadores e coletores. Possui no total 13 veículos, e 7 a disposição da população, sendo estes, 3 caminhões papa lixo e 4 caminhões disponibilizados pela SEMINF, que não são apropriados para o uso da limpeza pública.

Foi observado que a COLIMP, não possui uma estrutura que possa oferecer serviços adequados, deixando a desejar, principalmente, nas áreas periféricas da cidade, gerando insatisfação dos moradores dessas áreas. A mesma não oferece o serviço de coleta seletiva a esses moradores. A COLIMP possui muitos déficits tanto na prestação de serviços, quanto na sua estrutura organizacional. Muitos dos fatores que colaboram para esse mal funcionamento se deve à forma em que o gestor público utiliza os recursos do município, trazendo difusões na divisão de materiais entre as secretárias.

\section{III.3 ATIVIDADES EXERCIDAS PELA COLIMP}

Atualmente a COLIMP presta serviços a quase todo o município de Alenquer, com exceção de alguns bairros afastados devido à dificuldade na infraestrutura do município, e com exceção também das zonas rurais, que não recebe serviço de limpeza devido à falta de apoio e de recursos materiais que deviam ser advindos da prefeitura, fazendo com que esse tipo de serviço não chegue a essas comunidades.

Com os recursos disponibilizados pela prefeitura, atualmente são prestados serviços de coleta nas residências, de varredura nas vias públicas, serviços de roçagem, e limpeza de bueiros. Os trabalhos exercidos pelos coletores são exercidos de segunda feira à sexta feira, com início às seis horas da manhã, 
passando pelas áreas onde é possível ter acesso dos veículos utilizados pela COLIMP.

As atividades exercidas pelos varredores acontecem também de segunda feira à sexta feira, com exceção quando há atividade de grande público ao fim de semana em vias públicas. $\mathrm{O}$ trabalho realizado por estes tem início às sete e quinze da manhã. Os serviços de roçadores e limpadores de bueiros, acontecem quando há a necessidade. Principalmente na época chuvosa, onde é mais requisitado os serviços desses trabalhadores, pois há uma grande locomoção de resíduos e acúmulo de lama em direção aos bueiros e galerias, e também o crescimento de matagal em vias públicas, principalmente em bairros mais afastados, onde não há urbanização e pavimentação de ruas. Cada equipe de trabalhadores é monitorada por um chefe de turma, que é responsável por dar andamento e supervisionar o serviço exercido pelos garis.

A COLIMP conta com os serviços do coordenador, responsável por pensar nas atividades futuras e tomar decisões dentro da coordenadoria, auxiliado por dois secretários responsáveis por dar suporte nas decisões, andamento nos projetos e fazer relatórios sobre os serviços prestados pela coordenadoria. Entretanto, a falta de uma diferenciação organizacional, tanto a descentralização das tarefas fica comprometida.

Em decorrência de uma cultura clientelista internalizada pelos gestores locais, os cargos de diversos níveis de hierarquia, são indicados e nomeados deixando de lado indivíduos com maior capacidade analítica que importante para efetivo exercício de um cargo público, fato que produz disfunções em diversas áreas da estrutura organizacional da prefeitura de Alenquer.

Com essa cultura há excesso de cargos, implicando gastos à prefeitura e falta de controle pelo gestor público. Assim como em diversos departamentos da prefeitura a Coordenadoria de limpeza pública, não possui um líder com capacidades necessárias para exercer um trabalho que venha a operar de forma racional as atividades exercidas por seus subordinados, culminando na em conflitos entre gestor e seus funcionários.

Apesar de possuir duplicidade de cargos, a COLIMP não apresenta uma estrutura departamentalizada que possa favorecer a entrega dos serviços básicos, muito menos ampliar a oferta de serviços diferenciados como a coleta seletiva a esses moradores. A falta dessa estrutura amplia os déficits na prestação de serviços, bem como ampliar os gastos e a possibilidade de conflitos trazendo difusões na divisão de materiais entre as secretárias.

Dentre outros problemas enfatizados pelo entrevistado X, há a falta de cumprimento dos horários de trabalho, o consumo de drogas lícitas e ilícitas por alguns funcionários, ao ponto de alguns destes trabalharem sob o efeito de bebidas alcoólicas. Em decorrência disso amplia-se os conflitos organizacionais e como resultado a entrega dos serviços públicos ficam comprometidos.

Segundo o entrevistado $\mathrm{Y}$ alguns desses funcionários almejam largar o vício, porém não há um acompanhamento profissional que dê suporte a estes trabalhadores. Daí se nota uma falta integração horizontal entre as secretárias do município, que vem a ser um ponto fundamental para trazer uma melhor qualidade de vida dos funcionários, pois os mesmos não têm um suporte de outros setores. A integração horizontal entre os diferentes setores organizacionais amplia a efetividade na prestação de serviços.

É notório que entre os diferentes departamentos da prefeitura de Alenquer não há unidades interligadas, já que não se tem uma ajuda mútua entre os diferentes setores. Em função disso a coordenação organizacional é falida. Em função dessa frágil coordenação há um descaso com a saúde dos funcionários. Uma vez que o trabalho exercido por estes possui grandes riscos à segurança humana, pois estes funcionários correm risco de adquirir enfermidades pela exposição a vetores de contaminação e de lesões.

De acordo com o entrevistado $\mathrm{W}$ a falta de equipamentos apropriados para a execução de uma coleta de lixo amplia a insegurança humana dos garis. Dentre esses equipamentos estão, bonés, luvas, botas, máscaras e uniforme que os identifique. Notase uma ausência de todos esses materiais. Há também a ausência de outros materiais, como, enxada, vassouras, pás, roçadeiras e outros materiais, com isso limitando as tarefas a serem desenvolvidas com excelência. Para mitigar esses problemas sugere-se um organograma para nortear as ações desta coordenadoria, figura 2 .

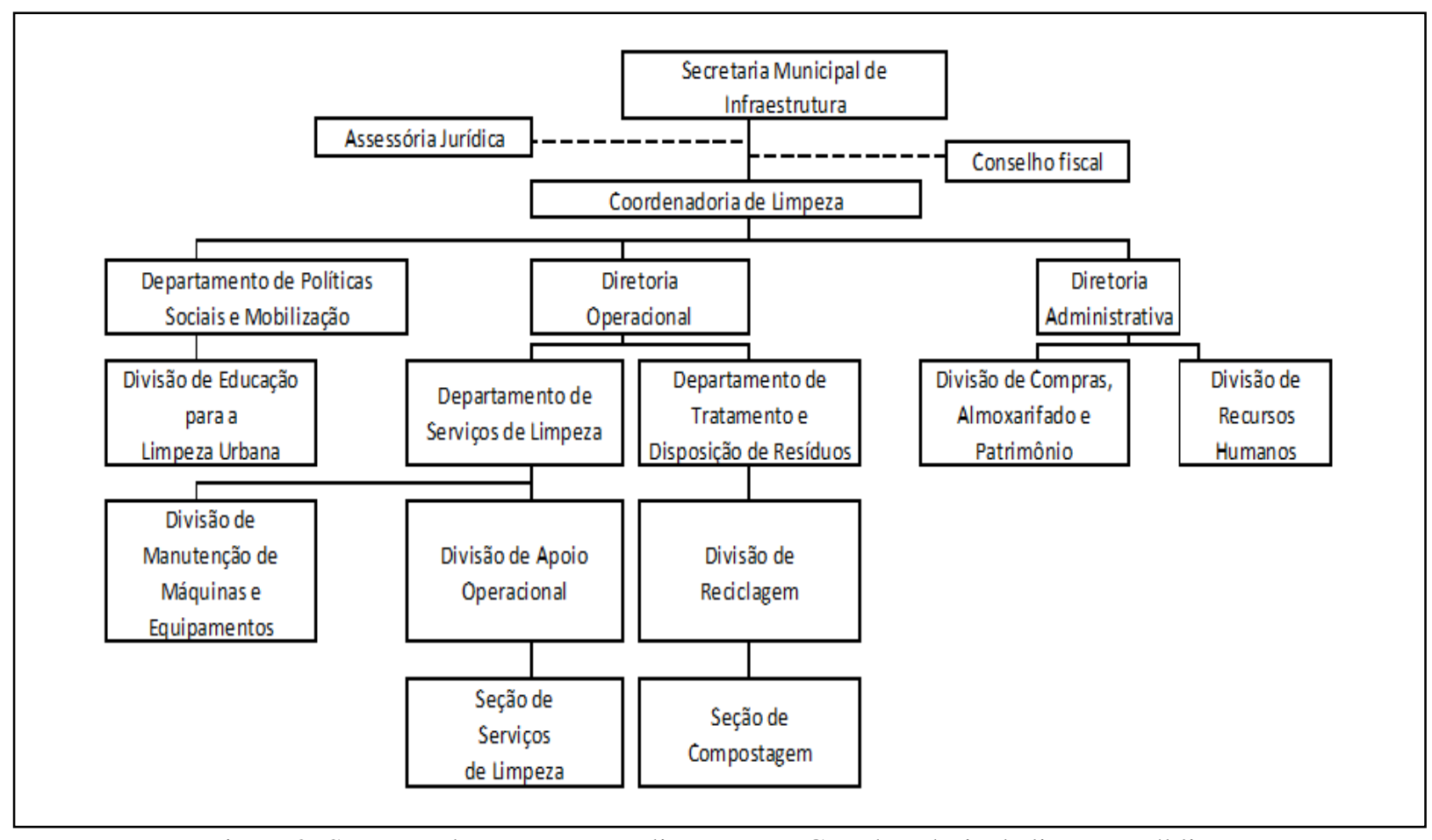

Figura 2: Sugestão de Departamentalização para Coordenadoria de limpeza pública. Fonte: Autores, (2019). 
Apesar dessa sugestão poder contribuir com a melhora dos trabalhos da coordenadoria, há outros problemas maiores que impactam na efetividade das organizações modernas e que pelas entrevistas ficaram visíveis. O desafio da COLIMP, como constituir agrupamentos humanos racionais e eficientes ao mesmo tempo que produz efeitos indesejados. Ou seja, como coordenar os esforços humanos a serviço dos fins da organização.

\section{CONSIDERAÇÕES FINAIS}

Este trabalho de pesquisa foi desenvolvido para entender como as interações intraorganizacionais da COLIMP influenciam a efetividade dos serviços prestados à comunidade externa e interna. $\mathrm{O}$ trabalho teve $\mathrm{o}$ intuito de identificar falhas organizacionais que devem ser mitigadas para que haja um melhor funcionamento deste departamento governamental.

De forma clara e objetiva podemos observar que a coordenadoria vem sofrendo um abandono por parte do poder público. Sendo as interações intraorganizacionais uma temática de importante para entender a efetividade dos serviços públicos, constatou-se que, no caso da coordenadoria de limpeza pública em Alenquer-PA, há déficits em várias áreas, sobretudo no que diz respeito a coordenação horizontal. Tais problemas evidenciam o somatório de um conjunto de fatores ligados a baixa capacidade dos atores ocupantes dos cargos de chefia.

Então constata-se que a falta de coordenação enfrentada por esta coordenadoria é oriunda da falta dos gestores superiores da prefeitura. Essa falta de suporte acaba por influenciar nos serviços prestados à população, mas também acaba impactando nos níveis de segurança humana dos funcionários. A insegurança é mais evidente nos servidores de rua, ou seja, os garis, pois observa-se que o descaso com estes é visível pela falta de condições adequadas de trabalho.

Com base nas entrevistas e nos constructos teóricos pode-se concluir que as interações intragovernamentais no município de Alenquer-PA são falhas, visto que a estrutura organizacional não comporta profissionais com capacidades para resolver as imprevisibilidades do ambiente. Assim, através desse levantamento preliminar podemos observar como a baixa integração intraorganizacional do setor público pode tornar a entrega de bens e serviços públicos ineficaz, uma vez que foi possível identificar as disfunções decorrentes da baixa integração horizontal dos departamentos e setores públicos. O desafio que fica é entender como coordenar os esforços humanos a serviço dos fins da organização de modo a torná-los mais eficiente.

\section{REFERÊNCIAS}

[1] Anne, T. (2015). The dilemmas of organisational capacity. Policy and Society, 34(3-4), 209-217. https://doi.org/10.1016/j.polsoc.2015.09.004

[2] Annosi, M. C., \& Brunetta, F. (2018). Resolving the dilemma between team autonomy and control in a post-bureaucratic era: Evidences from a telco multinational company. Organizational Dynamics. https://doi.org/10.1016/j.orgdyn.2018.03.002

[3] Atlas. (2010). Atlas do Desenvolvimento Humano no Brasil. Retrieved November 20, 2016, from http://www.atlasbrasil.org.br/2013/

[4] Bachrach, P., \& Baratz, M. S. (2011). Duas faces do poder. Revista de Sociologia E Política, 19(40), 149. Retrieved from http://search.proquest.com/openview/00832cad960ed4e41818707 0f4d254f5/1?pq-origsite $=$ gscholar

[5] Barbosa, K. G. N., \& Barbosa, A. C. N. (2014). O Impacto do lixo na saúde e a problemática da destinação final e coleta seletiva dos resíduos sólidos. polêm!ca, 13(3), 1372-1383. https://doi.org/10.12957/polemica.2014.11669

[6] Bardin, L. (1977). Análise de Conteúdo (70th ed.). Lisboa: Persona.

[7] Baumgartner, F. R., \& Jones, B. D. (1991). Agenda Dynamics and Policy Subsystems. The Journal of Politics, 53(4), 1044-1074. https://doi.org/10.2307/2131866

[8] Birkland, T. A. (2006). 5 Agenda Setting in Public Policy. Handbook of Public Policy Analysis, 63. Retrieved from https://books.google.com.br/books?hl=pt-

$\mathrm{BR} \& \mathrm{lr}=\& \mathrm{id}=\mathrm{vAbOBQAAQBAJ} \& \mathrm{oi}=\mathrm{fnd} \& \mathrm{pg}=\mathrm{PA} 63 \& \mathrm{dq}=$ Agend a+Setting+in+Public+Policy\&ots=QqK-

1f_AOx\&sig=bOeEd_bpml6myaRfoeCTTy5NiZM

[9] Brasil. (2006). Fundação Nacional de Saúde: Manual de Saneamento. Brasília: FUNASA.

[10] Cingolani, L. (2013). The State of State Capacity: a review of concepts, evidence and measures. MERIT Working Papers. Retrieved

https://ideas.repec.org/p/unm/unumer/2013053.html

[11] Cunha, M. P. (1999). Estrutura organizacional : dimensoes, determinantes e formatos. In M. P. Cunha (Ed.), Teoria organizacional: perspectivas e prospectivas. (pp. 251-279). Lisboa: Dom Quixote.

[12] Drexhage, J., \& Murphy, D. (2010). Sustainable development: from Brundtland to Rio 2012. Background paper prepared for consideration by the High Level Panel on Global Sustainability at its first meeting 19 September 2010., (September 2010), 26. Retrieved from https://www.popline.org/node/216968

[13] Gala, P. (2003). A Teoria Institucional de Douglas North. Revista de Economia Política, São Paulo.

[14] Gil, A. C. (2002). Como elaborar projetos de pesquisa. Como Elaborar Projetos de Pesquisa. https://doi.org/10.1111/j.14388677.1994.tb00406.x

[15] Gold, A. H., Malhotra, A., \& Segars, A. H. (2001). Knowledge Management: An Organizational Capabilities Perspective. Journal of Management Information Systems, 18(1), 185-214. https://doi.org/10.1080/07421222.2001.11045669

[16] Greenme. (2015). Má gestão do lixo causa problemas de saúde à toda sociedade. Retrieved April 24, 2018, from https://www.greenme.com.br/informar-se/lixo-e-reciclagem/2421ma-gestao-do-lixo-causa-problemas-de-saude-a-toda-sociedade

[17] Howlett, M., \& Ramesh, M. (2016). Achilles' heels of governance: Critical capacity deficits and their role in governance failures. Regulation and Governance, 10(4), 301-313. https://doi.org/10.1111/rego.12091 
[18] JONES, G. R. (2010). Teoria das organizações. São Paulo: Pearson.

[19] King, G., \& Murray, C. J. (2001). Rethinking human security. Political Science Quarterly, 116(4), 585-610.

[20] Korine, H. (1999). The new team organization:: Learning to manage arbitrariness. European Management Journal, 17(1), 1-7. https://doi.org/10.1016/S0263-2373(98)00055-3

[21] Kraatz, M. S. (1998). Learning by Association? Interorganizational Networks and Adaptation to Environmental Change. Academy of Management Journal, 41(6), 621-643. https://doi.org/10.5465/256961

[22] MELO, J. (2017). Descarte de pilhas e baterias: uma preocupação socio ambiental do município de Alenquer-PA. Universidade Federal do Oeste do Pará.

[23] Pires, R. R. C. (2009). Estilos de implementação e resultados de políticas públicas: fiscais do trabalho e o cumprimento da lei trabalhista no Brasil. Dados, 52(3), 734-769. https://doi.org/10.1590/S0011-52582009000300006

[24] PNUD. (1994). Human Development Report. New York: Oxford University Press. Problems. (2010, June). Problems of Policy Implementation. Retrieved October 17, 2016, from http://www.healthknowledge.org.uk/public-health-

textbook/medical-sociology-policy-economics/4c-equality-equitypolicy/problems-policy-implementation

[25] Robèrt, K.-H., Schmidt-Bleek, B., Aloisi de Larderel, J., Basile, G., Jansen, J. L., Kuehr, R., ... Wackernagel, M. (2002). Strategic sustainable development - selection, design and synergies of applied tools. Journal of Cleaner Production, 10(3), 197-214. https://doi.org/10.1016/S0959-6526(01)00061-0

[26] ROSÁRIO, E. M. F. DO. (2016). Abordagem da educação ambiental: lixo e reciclagem nas escolas no município de AlenquerPA. Trabalho de Conclusão de Curso, 42 p. Alenquer. Universidade Federal do Oeste do Pará.

[27] SANTOS, G. F. R. DOS. (2017). A ausênica de políticas públicas no município de Alenquer: como romper o paradigma da estagnação que impede o crescimento socioeconomico nos últimos 8 anos. Trabalho de Conclusão de Curso, 35 p. Alenquer-PA. Universidade Federal do Oeste do Pará.

[28] Stoner, J. A. F., \& FREEMAN, R. E. (1999). Administração. (: Phb Prentice/ Hall do Brasil, Ed.) (2 edição). Rio de Janeiro: LTC.

[29] Thomas, H. C. (2006). Trade Reforms and Food Security: Country Case Studies and Synthesis. Food \& Agriculture Org.

[30] Tsai, W. (2001). Knowledge Transfer in Intraorganizational Networks: Effects of Network Position and Absorptive Capacity on Business Unit Innovation and Performance. Academy of Management Journal, 44(5), 996-1004. https://doi.org/10.5465/3069443

[31] Unep. (2012). The business case for the green economicy. Retrieved February 1, 2015, from
http://www.unep.fr/shared/publications/pdf/DTIx1553xPABusinessCaseforGreenEconomy.pdf

[32] Unep. (2014). Managing and Conserving the Natural Resource Base for Sustained Economic and Social Development. Retrieved January 31, 2015, from http://www.unep.fr/shared/publications/pdf/DTIx1553xPABusinessCaseforGreenEconomy.pdf

[33] Vasconcellos, E., \& Hemsley, J. R. (1997). Estrutura das organizações: estruturas tradicionais, estruturas para a inovação, estrutura matricial (2 edição). Rio de Janeiro: Pioneira Thomson Learning. 